\title{
AUSLANDER ALGEBRAS AS QUASI-HEREDITARY ALGEBRAS
}

\author{
VLASTIMIL DLAB AND CLAUS MICHAEL RINGEL
}

The notion of a quasi-hereditary algebra has been introduced by E. Cline, B. Parshall and L. Scott $[7,2,5]$ in order to describe the so-called highest weight categories arising in the representation theory of Lie algebras and algebraic groups. Quasi-hereditary algebras are defined by the existence of a suitable chain of ideals, and the finite dimensional hereditary algebras are typical examples. In [3], also finite dimensional algebras of global dimension 2 are shown to be quasi-hereditary. Thus, the Auslander algebras are quasi-hereditary. Recall that the Auslander algebras $A$ can be constructed in the following way. Let $R$ be a representation-finite finite dimensional algebra; then $A$ is the endomorphism algebra $\operatorname{End}\left(M_{R}\right)$, where $M$ is a finite dimensional $R$-module such that every indecomposable $R$-module is isomorphic to a direct summand of $M$. We are going to introduce the notion of a splitting filtration on the class of all indecomposable $R$-modules and show that in this way we obtain a heredity chain of ideals of $A$ (see the definition below). Usually, there exist many splitting filtrations for a given $R$. Examples of splitting filtrations can be obtained from the Rojter measure, used by A. V. Rojter in his proof of the first Brauer-Thrall conjecture [6, or from the preprojective and preinjective partitions, introduced by M. Auslander and S. Smalø in [1].

Instead of dealing with finite dimensional algebras, we shall consider, more generally, semiprimary rings. Recall that an associative ring $A$ with 1 is called semiprimary provided that its Jacobson radical $N$ is nilpotent and $A / N$ is semisimple artinian. We say that an ideal $J$ of $A$ is a heredity ideal of $A$ if $J^{2}=J, J N J=0$ and $J$, considered as a right ideal, is a projective $A$-module. Following [2], a semiprimary ring $A$ is said to be quasi-hereditary provided that there exists a chain

$$
0=J_{0} \subseteq J_{1} \subseteq \ldots \subseteq J_{t-1} \subseteq J_{t} \subseteq \ldots \subseteq J_{m}=A
$$

of ideals of $A$ such that, for each $1 \leqslant t \leqslant m$, the ideal $J_{t} / J_{t-1}$ is a heredity ideal of $A / J_{t-1}$. Such a sequence of ideals is called a heredity chain. Some elementary facts related to these concepts can be found in [3].

\section{Splitting filtrations}

Let $R$ be a semiprimary ring of finite representation type. (Of course, this implies that $R$ is both left artinian and right artinian.) We denote by ind $R$ a complete set of representatives of the isomorphism classes of indecomposable modules. If $\mathscr{M} \subseteq$ ind $R$, then add $\mathscr{M}$ denotes the full additive subcategory of $\bmod R$ generated by $\mathscr{M}$. Its objects are direct sums of copies of modules in $\mathscr{M}$. A direct sum of $a$ copies of a module $X$ will be denoted by $a X$.

Received 5 January 1988; revised 21 June 1988.

1980 Mathematics Subject Classification (1985 Revision) 16A46. 
Definition. A chain $\varnothing=\mathscr{M}_{0} \subseteq \mathscr{M}_{1} \subseteq \ldots \subseteq \mathscr{M}_{m}=$ ind $R$ of subsets of ind $R$ is called a splitting filtration provided that the following two properties are satisfied for $1 \leqslant t \leqslant m$.

(1) If $M, M^{\prime} \in \mathscr{M}_{t}$, and $\phi: M \rightarrow M^{\prime}$ is not invertible, then $\phi$ factors through a module in add $\mathscr{M}_{t-1}$.

(2) If $0 \rightarrow U \stackrel{\phi}{\rightarrow} W \stackrel{\psi}{\rightarrow} V \rightarrow 0$ is an exact sequence of $R$-modules, with $W \in$ add $\mathscr{M}_{t}$, and if $M$ is a module in $\mathscr{M}_{t} \backslash \mathscr{M}_{t-1}$, then either

(a) $U \in$ add $\mathscr{M}_{t}$, and there is a decomposition $U=a M \oplus U^{\prime}$ with $a \geqslant 0$ such that $M$ is not a direct summand of $U^{\prime}$ and such that

$$
a M \stackrel{\left[\begin{array}{l}
1 \\
0
\end{array}\right]}{\longrightarrow} a M \oplus U^{\prime}=U \stackrel{\phi}{\longrightarrow} W
$$

is a split monomorphism, or else

(b) $V \in$ add $\mathscr{M}_{t}$, and there is a decomposition $V=b M \oplus V^{\prime}$ such that $M$ is not a direct summand of $V$, and such that

$$
W \stackrel{\psi}{\longrightarrow} V=b M \oplus V^{\prime} \stackrel{[10]}{\longrightarrow} b M
$$

is a split epimorphism.

We shall see below that there are usually many splitting filtrations for a given $R$. Note that the concept of a splitting filtration is self-dual. If $\mathscr{M}_{0} \subseteq \mathscr{M}_{1} \subseteq \ldots \subseteq \mathscr{M}_{m}$ is a splitting filtration of ind $R$, and $(\bmod R)^{o p}$ is identified with a module category $\bmod R^{\prime}$ (where $R^{\prime}=$ End $\left(Q_{R}\right)^{o p}$ for some finitely generated injective cogenerator $Q_{R}$ ), then $\mathscr{M}_{0}^{o p} \subseteq \mathscr{M}_{1}^{o p} \subseteq \ldots \subseteq \mathscr{M}_{m}^{o p}$ is a splitting filtration of ind $R^{\prime}$. We shall use the following criterion for verifying that a chain of subsets of ind $R$ is a splitting filtration.

Proposition 1. Let $\varnothing=\mathscr{M}_{0} \subseteq \mathscr{M}_{1} \subseteq \ldots \subseteq \mathscr{M}_{m}=$ ind $R$ be a chain of subsets of ind $R$ such that the following two properties are satisfied for all $1 \leqslant t \leqslant m$.

$\left(1^{\prime}\right)$ The modules in $\mathscr{M}_{t} \backslash \mathscr{M}_{t-1}$ are of equal length.

$\left(2^{\prime}\right)$ Every monomorphism $X \rightarrow Y$, where $Y \in$ add $\mathscr{M}_{t}$ and $X \in \mathscr{M}_{t} \backslash \mathscr{M}_{t-1}$, splits. Then, $\mathscr{M}_{0} \subseteq \mathscr{M}_{1} \subseteq \ldots \subseteq \mathscr{M}_{m}$ is a splitting filtration.

Proof. We assume that the properties $\left(1^{\prime}\right)$ and $\left(2^{\prime}\right)$ are satisfied. First, we show that add $\mathscr{M}_{t}$ is closed under submodules. For, let $X$ be an indecomposable submodule of $Y$, where $Y \in \operatorname{add} \mathscr{M}_{t}$. Let $X \in \mathscr{M}_{s} \backslash \mathscr{M}_{s-1}$, and assume that $s>t$. Since $\mathscr{M}_{t} \subseteq \mathscr{M}_{s}$, it follows from (2') that the inclusion $X \rightarrow Y$ splits, thus $X$ belongs to $\mathscr{M}_{t} \subseteq \mathscr{M}_{s-1}$; a contradiction.

In order to verify (1), let $\phi: M \rightarrow M^{\prime}$ be a non-invertible map, with $M, M^{\prime}$ in $\mathscr{M}_{t} \backslash \mathscr{M}_{t-1}$. Let $I$ be the image of $\phi$. Since $M, M^{\prime}$ have equal length, and $\phi$ is not invertible, $I$ is a proper submodule. As we have seen above, $I \in \operatorname{add} \mathscr{M}_{t}$. Let $I^{\prime}$ be an indecomposable direct summand of $I$. Then $I^{\prime} \in \mathscr{M}_{t-1}$, since otherwise the inclusion $I^{\prime} \rightarrow M^{\prime}$ would split. This shows that $I \in$ add $\mathscr{M}_{t-1}$.

Before we verify (2), let us show, by induction, that $\left(2^{\prime}\right)$ implies the following stronger assertion. If $Y \in$ add $\mathscr{M}_{t}$ and $X \in \mathscr{M}_{t} \backslash \mathscr{M}_{t-1}$, then the monomorphism $a X \rightarrow Y$, $a \geqslant 1$ splits. Indeed, assume that $\mu: a X \rightarrow Y$ is such a monomorphism and write $\mu=\left[\mu_{1}, \mu_{2}\right]:(a-1) X \oplus X \rightarrow Y$. By induction, $\mu_{1}$ splits; hence, up to isomorphism,

$$
\mu=\left[\begin{array}{ll}
1 & \mu_{12} \\
0 & \mu_{22}
\end{array}\right]:(a-1) X \oplus X \longrightarrow(a-1) X \oplus Y^{\prime} \simeq Y .
$$


It follows that $\mu_{22}$ is a monomorphism $X \rightarrow Y^{\prime}$ and $Y^{\prime} \in$ add $\mathscr{M}_{t}$. Thus, according to $\left(2^{\prime}\right), \mu_{22}$ splits: $\rho \mu_{22}=1$ for some $\rho: Y^{\prime} \rightarrow X$. Then

$$
\left[\begin{array}{cc}
1 & -\mu_{12} \rho \\
0 & \rho
\end{array}\right]:(a-1) X \oplus Y^{\prime} \longrightarrow(a-1) X \oplus X
$$

is a retraction for $\mu$.

Now, to show the validity of (2), let $0 \rightarrow U \stackrel{\phi}{\rightarrow} W \stackrel{\psi}{\rightarrow} V \rightarrow 0$ be exact and $W \in \operatorname{add} \mathscr{M}_{t}$. Choose $M \in \mathscr{M}_{t} \backslash \mathscr{M}_{t-1}$. We claim that (a) is satisfied. Since add $\mathscr{M}_{t}$ is closed under submodules, $U \in \operatorname{add} \mathscr{M}_{t}$. Decompose $U=a M \oplus U^{\prime}$ so that $M$ is not a direct summand of $U^{\prime}$. The map

$$
a M \stackrel{\left[\begin{array}{l}
1 \\
0
\end{array}\right]}{\longrightarrow} a M \oplus U^{\prime}=U \stackrel{\phi}{\longrightarrow} W
$$

is a monomorphism, and thus it splits according to the preceding assertion.

For the convenience of the reader, we state the dual result.

Proposition $1^{*}$. Let $\varnothing=\mathscr{M}_{0} \subseteq \mathscr{M}_{1} \subseteq \ldots \subseteq \mathscr{M}_{m}=$ ind $R$ be a chain of subsets of ind $R$, such that the following two properties are satisfied for all $1 \leqslant t \leqslant m$.

$\left(1^{\prime}\right)$ The modules in $\mathscr{M}_{t} \backslash \mathscr{M}_{t-1}$ are of equal length.

$\left(2^{\prime *}\right)$ Every epimorphism $Y \rightarrow Z$, where $Y \in$ add $\mathscr{M}_{t}$ and $Z \in \mathscr{M}_{t} \backslash \mathscr{M}_{t-1}$, splits. Then, $\mathscr{M}_{0} \subseteq \mathscr{M}_{1} \subseteq \ldots \subseteq \mathscr{M}_{m}$ is a splitting filtration.

In the remainder of this section, let us exhibit some examples of splitting filtrations.

The Rojter filtration. In his proof of the first Brauer-Thrall conjecture for finite dimensional algebras, A. V. Rojter [6] used a specific ordering of the indecomposable modules. This procedure (or rather its dual) was formalized by P. Gabriel [4] by introducing the notion of the Rojter measure $\rho(X)$ for any object in a length category: $\rho(X)=\max \left\{l\left(X_{1}\right), l\left(X_{2}\right), \ldots, l\left(X_{n}\right)\right\}$, where $l\left(X_{i}\right)$ denotes the length of the object $X_{i}$ and the maximum is taken over all chains $0 \subset X_{1} \subset X_{2} \subset \ldots \subset X_{n} \subseteq X$ of indecomposable subobjects $X_{i}$ of $X$ with respect to the following (linear) order defined on the finite subsets of the set of all positive integers. If $K \neq L$ are two such subsets, $K<L$ if and only if the least natural number in $(K \cup L) \backslash(K \cap L)$ belongs to $L$. Given a ring $R$, let $\rho_{1}<\rho_{2}<\ldots<\rho_{m}$ be all possible Rojter measures of (indecomposable) $R$-modules, and let $\mathscr{M}_{t}$ be the set of all modules $M$ in ind $R$ with $\rho(M) \leqslant \rho_{t}, 1 \leqslant t \leqslant m$. By the definition of the Rojter measure, indecomposable modules with the same Rojter measure are of equal length. By [4, Proposition 5.2], the condition (2') is satisfied. Thus $\varnothing \subset \mathscr{M}_{1} \subset \mathscr{M}_{2} \subset \ldots \subset \mathscr{M}_{m}$ is a splitting filtration. Let us add that the modules in $\mathscr{M}_{1}$ are just all simple modules.

The dual Rojter filtration. Given an $R$-module $M$, let $\rho^{*}(M)$ be the Rojter measure of $M$ considered as an object of $(\bmod R)^{o p}$. (Whereas $\rho(M)$ measures the existence of indecomposable submodules of $M$, the dual Rojter measure $\rho^{*}(M)$ measures the existence of indecomposable factor modules of $M$.) Let $\rho_{1}^{*}<\ldots<\rho_{m^{*}}^{*}$ be the possible dual Rojter measures $\rho^{*}(M)$ of (indecomposable) $R$-modules $M$. Let $\mathscr{M}_{i}^{*}$ be the set of modules $M$ in ind $R$ with $\rho^{*}(M) \leqslant \rho_{t}^{*}$. Then

$$
\varnothing \subset \mathscr{M}_{1}^{*} \subset \mathscr{M}_{2}^{*} \subset \ldots \subset \mathscr{M}_{m^{*}}^{*}
$$

is a splitting filtration, and again, $\mathscr{M}_{1}^{*}$ is the set of all simple modules. 
The filtrations corresponding to the preprojective partition. Let $\mathscr{P}_{0}, \mathscr{P}_{1}, \ldots, \mathscr{P}_{p}$ be the preprojective partition of ind $R$, as introduced by Auslander and Smalø [1]. Given an indecomposable module $M$, let $\pi(M)$ be defined by $M \in \mathscr{P}_{\pi(M)}$. Let

$$
\varnothing=\mathscr{M}_{0}^{p} \subseteq \mathscr{M}_{1}^{p} \subseteq \ldots \subseteq \mathscr{M}_{m^{\prime}}^{p}=\text { ind } R
$$

be a chain of subsets of ind $R$ such that for $1 \leqslant t \leqslant m^{\prime}$, the modules in $\mathscr{M}_{t}^{p} / \mathscr{M}_{t-1}^{p}$ have equal length, and such that for $M \in \mathscr{M}_{s}^{p} \backslash \mathscr{M}_{s-1}^{p}, N \in \mathscr{M}_{t}^{p} \backslash \mathscr{M}_{t-1}^{p}$, with $s<t$, we have $\pi(M) \geqslant \pi(N)$. The defining property of the preprojective partition shows that the condition $\left(2^{\prime *}\right)$ is satisfied, thus $\mathscr{M}_{0}^{p} \subseteq \mathscr{M}_{1}^{p} \subseteq \ldots \subseteq \mathscr{M}_{m}^{p}$ is a splitting filtration.

The filtrations corresponding to the preinjective partition. These are defined in the same way. Again, we use the ordering given by this partition, and refine it in order that the length condition $\left(1^{\prime}\right)$ is satisfied.

Let us remark that these four kinds of filtrations are, in general, distinct. Consider a hereditary algebra of type $\mathbb{A}_{3}$ with one simple projective and two simple injective modules: its Auslander-Reiten quiver is of the following form.

The Rojter filtration is

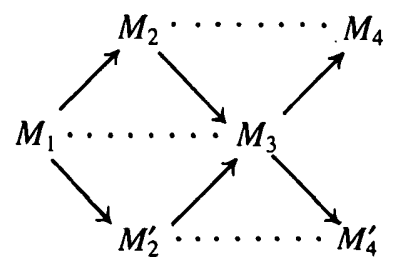

$$
\varnothing \subset\left\{M_{1}, M_{4}, M_{4}^{\prime}\right\} \subset\left\{M_{1}, M_{2}, M_{2}^{\prime}, M_{4}, M_{4}^{\prime}\right\} \subset \text { ind } R,
$$

and the dual Rojter filtration is

$$
\varnothing \subset\left\{M_{1}, M_{4}, M_{4}^{\prime}\right\} \subset\left\{M_{1}, M_{3}, M_{4}, M_{4}^{\prime}\right\} \subset \text { ind } R ;
$$

there are several filtrations corresponding to the preprojective partition $\mathscr{P}_{0}=\left\{M_{1}, M_{2}, M_{2}^{\prime}\right\}, \mathscr{P}_{1}=\left\{M_{3}\right\}, \mathscr{P}_{2}=\left\{M_{4}, M_{4}^{\prime}\right\} ;$ for example,

$$
\varnothing \subset\left\{M_{4}, M_{4}^{\prime}\right\} \subset\left\{M_{3}, M_{4}, M_{4}^{\prime}\right\} \subset\left\{M_{1}, M_{3}, M_{4}, M_{4}^{\prime}\right\} \subset \text { ind } R ;
$$

and several filtrations corresponding to the preinjective partition $\mathscr{I}_{0}=\left\{M_{3}, M_{4}, M_{4}^{\prime}\right\}$, $\mathscr{I}_{1}=\left\{M_{2}, M_{2}^{\prime}\right\}, \mathscr{I}_{2}=\left\{M_{1}\right)$; for example,

$$
\varnothing \subset\left\{M_{1}\right\} \subset\left\{M_{1}, M_{2}, M_{2}^{\prime}\right\} \subset\left\{M_{1}, M_{2}, M_{2}^{\prime}, M_{3}\right\} \subset \text { ind } R .
$$

All these filtrations are distinct.

Given a splitting filtration $\mathscr{M}_{0} \subseteq \mathscr{M}_{1} \subseteq \ldots \subseteq \mathscr{M}_{m}$, it may or may not be the case that the subcategories add $\mathscr{M}_{t}, 0<t<m$, are closed under submodules, or under factor modules. In the case when a splitting filtration is given by Proposition 1 (such as the Rojter filtration, or any filtration derived from the preinjective partition), these subcategories are closed under submodules. Dually, in the case when a splitting filtration is given by Proposition 1* (such as the dual Rojter filtration, or any filtration derived from the preprojective partition), these subcategories are closed under factor modules. The example above (a hereditary algebra of type $A_{3}$ with one simple projective and two simple injective modules) shows that in general there will not exist any splitting filtration $\mathscr{M}_{0} \subseteq \mathscr{M}_{1} \subseteq \ldots \subseteq \mathscr{M}_{m}$ such that all add $\mathscr{M}_{t}$ are closed both under submodules and factor modules. 


\section{The main theorem}

As before, $R$ is a semiprimary ring of finite representative type. Let $A$ be the endomorphism ring End $(\oplus X)$ of the direct sum of all $X \in$ ind $R$. Thus $A$ is a basic Auslander algebra (and any basic Auslander algebra can be obtained in this way).

THEOREM. Let $\mathscr{M}_{0} \subseteq \mathscr{M}_{1} \subseteq \ldots \subseteq \mathscr{M}_{m}$ be a splitting filtration of ind $R$. Let $J_{t}$ be the set of all endomorphisms in $A$ which factor through a module in add $\mathscr{M}_{t}, 1 \leqslant t \leqslant m$. Then

$$
0=J_{0} \subseteq J_{1} \subseteq \ldots \subseteq J_{m}=A
$$

is a heredity chain of $A$.

Proof. Given $Y \in$ ind $R$, we denote by $e(Y)$ the endomorphism of $\oplus X$ which projects canonically $\oplus X$ onto $Y$. Thus, the elements $e(X), X \in$ ind $R$, form a set of pairwise orthogonal primitive idempotents of $A$. Of course, $A$ is basic: for different modules $X, Y$ in ind $R$, the idempotents $e(X)$ and $e(Y)$ are non-equivalent. Note that for $X, Y \in$ ind $R$, we can identify $e(X) A e(Y)$ with $\operatorname{Hom}_{R}(Y, X)$. If $N$ is the radical of $A, e(X) N e(Y)$ is the set of non-invertible maps from $Y$ to $X$. The ideal $J_{t}$ is generated by the set of all $e(X)$ with $X \in \mathscr{M}_{t}$; thus $J_{t}$ is an idempotent ideal. Let $1 \leqslant t \leqslant m$, and consider the residue ring $\bar{A}=A / J_{t-1}$. The residue class of $\alpha \in A$ will be denoted by $\bar{\alpha}$; a similar notation will be used for subsets of $A$. It will be convenient to denote $J_{t}$ by $J$, and write $e=\sum_{X \in \mathscr{M}_{t}} e(X)$; thus, $J=A e A$. Clearly, $\bar{J}$ is idempotent. Furthermore, note that $\bar{J} \bar{N} \bar{J}=0$. For, given $X, Y \in \mathscr{M}_{t}, e(X) N e(Y)$ is the set of non-invertible maps $Y \rightarrow X$, and these, by condition (1), belong to $J_{t-1}$. Hence, it remains to show that $\bar{J}_{\bar{A}}$ is projective, or, equivalently, that the multiplication map $\bar{A} \bar{e} \bigotimes_{\bar{e} \bar{A} \overline{\bar{e}}} \bar{e} \bar{A} \rightarrow \bar{A}$ is injective (cf. [3, Statement 7, Appendix]). Multiplying from the left by $\overline{e(X)}$ and from the right by $\overline{e(Y)}$, where $X, Y \in$ ind $R$, we note that the multiplication map is the direct sum of the corresponding multiplication maps $\overline{e(X)} \bar{A} \bar{e} \bigotimes_{\bar{e} \bar{A} \bar{e}} \bar{e} \bar{A} \bar{e}(Y) \rightarrow \overline{e(X)} \bar{A} \bar{e}(Y)$. Thus, it is sufficient to show that the multiplication map $\overline{e(X)} \bar{A} \bar{e} \bigotimes_{\bar{e} \bar{A} \bar{e}} \bar{e} \bar{A} \bar{e} \overline{(Y)} \rightarrow \bar{A}$ is injective. To this end, we fix $X, Y$ in ind $R$.

Let $\mathscr{M}_{t} \backslash \mathscr{M}_{t-1}=\left\{M_{1}, \ldots, M_{p}\right\}$ and $e_{i}=e\left(M_{i}\right)$; thus $\bar{e}=\sum_{i=1}^{p} \bar{e}_{i}$. Since we know that $\bar{J} \bar{J} \bar{J}=0$, it follows that $\bar{e} \bar{A} \bar{e}=\bigoplus_{i=1}^{p} \bar{e}_{i} \bar{A} \bar{e}_{i}$ and that $\bar{e}_{i} \bar{A}_{\bar{e}_{i}}$ are division rings. It follows that $\overline{e(X)} \bar{A} \bar{e} \otimes_{\bar{e} \bar{A} \bar{e}} \bar{e} \overline{A e(Y)}$ can be identified with $\bigoplus_{i=1}^{p} G_{i}$, where

$$
G_{i}=\overline{e(X)} \bar{A} \bar{e}_{i} \bigotimes_{\bar{e}_{i} \bar{A} \bar{e}_{i}} \bar{e}_{i} \overline{A e(Y)}
$$

Let $g_{i} \in G_{i}$, and assume that the element $\left(g_{i}, \ldots, g_{p}\right) \in \bigoplus_{i=1}^{p} G_{i}$ is mapped under the multiplication map to zero. The element $g_{i} \in G_{i}$ is of the form $g_{i}=\sum_{j=1}^{c_{i}} \bar{\alpha}_{i j} \otimes \bar{\beta}_{i j}$, with $\alpha_{i j} \in \operatorname{Hom}_{R}\left(M_{i}, X\right)$ and $\beta_{i j} \in \operatorname{Hom}_{R}\left(Y, M_{i}\right)$, for $1 \leqslant j \leqslant c_{i}$. Let

We put

$$
\alpha_{i}=\left[\alpha_{i 1} \ldots \alpha_{i c_{i}}\right]: c_{i} M_{i} \longrightarrow X, \quad \beta_{i}=\left[\begin{array}{c}
\beta_{i 1} \\
\vdots \\
\beta_{i c_{i}}
\end{array}\right]: Y \longrightarrow c_{i} M_{i}
$$

$$
C=\bigoplus_{i=1}^{p} c_{i} M_{i}, \quad \alpha=\left[\alpha_{1} \ldots \alpha_{p}\right]: C \longrightarrow X, \quad \beta=\left[\begin{array}{c}
\beta_{1} \\
\vdots \\
\beta_{p}
\end{array}\right]: Y \longrightarrow C
$$


The multiplication map sends $\left(g_{1}, \ldots, g_{p}\right)$ to the residue class of $\sum_{i=1}^{p} \sum_{j=1}^{c_{i}} \alpha_{i j} \beta_{i j}$ in $\bar{A}$. By our assumption, this residue class is zero, thus $\alpha \beta \in J_{t-1}$. This means that $\alpha \beta=\alpha^{\prime} \beta^{\prime}$, where $\alpha^{\prime}: C^{\prime} \rightarrow X, \beta^{\prime}: Y \rightarrow C^{\prime}$, with $C^{\prime} \in$ add $\mathscr{M}_{t-1}$. Consequently, there is a commutative diagram

such that the sequence

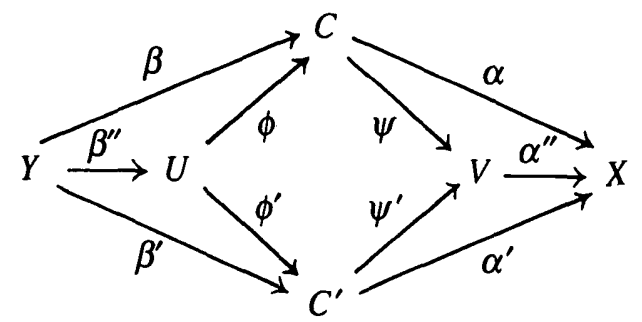

$$
0 \longrightarrow U \stackrel{\left[\begin{array}{l}
\phi \\
\phi^{\prime}
\end{array}\right]}{\longrightarrow} C \oplus C^{\prime} \stackrel{\left[\psi-\psi^{\prime}\right]}{\longrightarrow} V \longrightarrow 0
$$

is exact. Indeed, denote by $\left(U, \phi, \phi^{\prime}\right)$ the pullback of $\alpha, \alpha^{\prime}$, and by $\left(V, \psi, \psi^{\prime}\right)$ the pushout of $\phi, \phi^{\prime}$. We want to show that $g_{i}=0$ for each $1 \leqslant i \leqslant p$. Consider the exact sequence and the module $M_{i}$ : The condition (2) of a splitting filtration shows that one of the two assertions (a), (b) holds. Without loss of generality and, up to duality, we may assume that $i=1$, and that the assertion (a) holds. Thus, $U \in \operatorname{add} \mathscr{M}_{t}$, and there is a decomposition $U=a M_{1} \oplus U^{\prime}$, such that $M_{1}$ is not a direct summand of $U^{\prime}$, and such that for $\mu=\left[\begin{array}{l}1 \\ 0\end{array}\right]: a M_{1} \rightarrow a M_{1} \oplus U^{\prime}$, the map $\left[\begin{array}{l}\phi \\ \phi^{\prime}\end{array}\right] \mu: a M_{1} \rightarrow C \oplus C^{\prime}$ is a split monomorphism. Since $M_{1}$ is not a direct summand of $C^{\prime}$, also $\phi \mu: a M_{1} \rightarrow C$ is a split monomorphism. Now, $C=\bigoplus_{i=1}^{p} c_{i} M_{i}$, and we denote by $\pi_{i}: C \rightarrow c_{i} M_{i}$ the canonical projections. Since the $M_{i}$ are pairwise non-isomorphic, also $\pi_{1} \phi \mu$ is a split monomorphism and thus there is a retraction $\rho$ such that $\rho \pi_{1} \phi \mu=1_{a M_{1}}$. Let $\omega=\pi_{1} \phi \mu \rho$, and $\omega^{\prime}=1-\omega$; these are idempotent endomorphisms of $c_{1} M_{1}$.

We claim that $\omega^{\prime} \beta_{1}$ and $\alpha_{1} \omega$ belong to $J_{t-1}$. To furnish a proof, let

$$
\beta^{\prime \prime}=\left[\begin{array}{l}
\beta_{1}^{\prime \prime} \\
\beta_{2}^{\prime \prime}
\end{array}\right]: Y \longrightarrow a M_{1} \oplus U^{\prime} \quad \text { and } \quad \phi=\left[\phi_{1} \phi_{2}\right]: a M_{1} \oplus U^{\prime} \longrightarrow C ;
$$

thus $\phi_{1}=\phi \mu$. Then

since

$$
\omega^{\prime} \beta_{1}=\omega^{\prime} \pi_{1} \beta=\omega^{\prime} \pi_{1} \phi \beta^{\prime \prime}=\omega^{\prime} \pi_{1} \phi_{1} \beta_{1}^{\prime \prime}+\omega^{\prime} \pi_{1} \phi_{2} \beta_{2}^{\prime \prime}=\omega^{\prime} \pi_{1} \phi_{2} \beta_{2}^{\prime \prime},
$$

$$
\omega^{\prime} \pi_{1} \phi_{1} \beta_{1}^{\prime \prime}=(1-\omega) \pi_{1} \phi_{1} \beta_{1}^{\prime \prime}=\left(1-\pi_{1} \phi \mu \rho\right) \pi_{1} \phi \mu \beta_{1}^{\prime \prime}=\pi_{1} \phi \mu \beta_{1}^{\prime \prime}-\pi_{1} \phi \mu \rho \pi_{1} \phi \mu \beta_{1}^{\prime \prime}=0 .
$$

But $\pi_{1} \phi_{2}: U^{\prime} \rightarrow c_{1} M_{1}$ is not invertible and therefore factors through a module in add $\mathscr{M}_{t-1}$ by (1) of the definition of a splitting filtration. Hence $\omega^{\prime} \beta_{1}=\omega^{\prime} \pi_{1} \phi_{2} \beta_{2}^{\prime \prime}$ belongs to $J_{t-1}$. The first assertion is established.

To prove the second assertion, we note that

and thus

$$
\sum_{i=1}^{p} \alpha_{i} \pi_{i} \phi=\alpha \phi=\alpha^{\prime} \phi^{\prime}
$$

consequently,

$$
\alpha_{1} \pi_{1} \phi=-\sum_{i=2}^{p} \alpha_{i} \pi_{i} \phi+\alpha^{\prime} \phi^{\prime}
$$

$$
\alpha_{1} \omega=\alpha_{1} \pi_{1} \phi \mu \rho=\sum_{i=2}^{p} \alpha_{i} \pi_{i} \phi \mu \rho+\alpha^{\prime} \phi^{\prime} \mu \rho
$$


But $\pi_{i} \phi \mu: a M_{1} \rightarrow c_{i} M_{i}$ belongs to $J_{t-1}$ for $i \geqslant 2$, and $\alpha^{\prime} \phi^{\prime} \mu \rho$ factors through $C^{\prime} \in$ add $\mathscr{M}_{t-1}$, and thus it also belongs to $J_{t-1}$.

Now, we want to rewrite $g_{1}$ in order to see that $g_{1}=0$. This will be done using the following general lemma concerning tensor products of homomorphism spaces.

Lemma. Let $X, Y, M$ be modules and $c$ a positive integer. Let $\delta: Y \rightarrow c M$ and $\gamma: c M \rightarrow X$ be homomorphisms, $\omega$ an endomorphism of $c M$ with $\omega^{2}=\omega$, and define $\omega^{\prime}=1-\omega \in$ End $(c M)$. For $1 \leqslant j \leqslant c$, let $l_{j}: M \rightarrow c M$ be the jth inclusion, and $\varepsilon_{j}: c M \rightarrow M$ the jth projection. Then, in $\operatorname{Hom}(M, X) \otimes_{\mathrm{End}(M)} \operatorname{Hom}(Y, M)$, we have

$$
\sum_{j=1}^{c} \gamma l_{j} \otimes \varepsilon_{j} \delta=\sum_{j=1}^{c} \gamma \omega l_{j} \otimes \varepsilon_{j} \omega \delta+\sum_{j=1}^{c} \gamma \omega^{\prime} \iota_{j} \otimes \varepsilon_{j} \omega^{\prime} \delta
$$

Proof. First using that $\sum_{j=1}^{c} l_{j} \varepsilon_{j}=1_{c M}, \varepsilon_{j} \omega l_{s} \in \operatorname{End}(M)$ and $\omega^{2}=\omega$, we can rewrite the first term on the right of the equality as follows:

$$
\sum_{j=1}^{c} \gamma \omega l_{j} \otimes \varepsilon_{j} \omega \delta=\sum_{j=1}^{c} \gamma \omega l_{j} \otimes\left(\sum_{s=1}^{c} \varepsilon_{j} \omega l_{s} \varepsilon_{s} \delta\right)=\sum_{j, s} \gamma \omega l_{j} \varepsilon_{j} \omega l_{s} \otimes \varepsilon_{s} \delta=\sum_{s=1}^{c} \gamma \omega l_{s} \otimes \varepsilon_{s} \delta .
$$

Similarly, the second term can be rewritten as follows:

$$
\sum_{j=1}^{c} \gamma \omega^{\prime} \iota_{j} \otimes \varepsilon_{j} \omega^{\prime} \delta=\sum_{s=1}^{c} \gamma \omega^{\prime} \iota_{s} \otimes \varepsilon_{s} \delta
$$

Thus, since $\omega+\omega^{\prime}=1_{c M}$,

$$
\begin{aligned}
\sum_{j} \gamma \omega l_{j} \otimes \varepsilon_{j} \omega \delta+\sum_{j} \gamma \omega^{\prime} l_{j} \otimes \varepsilon_{j} \omega^{\prime} \delta & =\sum_{s=1}^{c} \gamma \omega \iota_{s} \otimes \varepsilon_{s} \delta+\sum_{s=1}^{c} j \omega^{\prime} l_{s} \otimes \varepsilon_{s} \delta \\
& =\sum_{s=1}^{c} \gamma\left(\omega+\omega^{\prime}\right) l_{s} \otimes \varepsilon_{s} \delta=\sum_{s=1}^{c} \gamma l_{s} \otimes \varepsilon_{s} \delta
\end{aligned}
$$

The lemma is established.

Now, we are ready to finish the proof that $g_{i}=0$. We use the lemma for $M=M_{1}$. As in the lemma, the inclusions $M_{1} \rightarrow c_{1} M_{1}$ are denoted by $l_{j}$, the projections $c_{1} M_{1} \rightarrow M_{1}$ by $\varepsilon_{j}, 1 \leqslant j \leqslant c_{1}$. In the tensor product

rewrite

$$
e(X) A e_{1} \otimes e_{e_{1} A e_{1}} A e(Y)=\operatorname{Hom}\left(M_{1}, X\right) \underset{\operatorname{End}\left(M_{1}\right)}{\bigotimes} \operatorname{Hom}\left(Y, M_{1}\right)
$$

$$
\sum_{j=1}^{c_{1}} \alpha_{1 j} \otimes \beta_{1 j}=\sum_{j=1}^{c_{1}} \alpha_{1} l_{j} \otimes \varepsilon_{j} \beta_{1}=\sum_{j=1}^{c_{1}} \alpha_{1} \omega l_{j} \otimes \varepsilon_{j} \omega \beta_{1}+\sum_{j=1}^{c_{1}} \alpha_{1} \omega^{\prime} l_{j} \otimes \varepsilon_{j} \omega^{\prime} \beta_{1} .
$$

Since $\alpha_{1} \omega$ and $\omega^{\prime} \beta_{1}$ belong to $J_{t-1}$, we see that both right-hand terms become zero in $\overline{e(X)} \bar{A} \bar{e}_{1} \otimes_{\bar{e}_{1} \bar{A} \bar{e}_{1}} \bar{e}_{1} \bar{A} e(Y)$. Thus $g_{1}=0$. The proof of the theorem is complete.

EXAMPLE. The following illustration of the theorem may be found useful. Let $R$ be the path algebra of

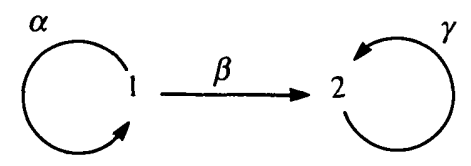


modulo the ideal generated by $\left\{\alpha^{2}, \alpha \beta-\beta \gamma, \gamma^{2}\right\}$. The algebra $R$ possesses nine indecomposable modules which may be easily displayed in the Auslander-Reiten quiver as follows (here the two vertical dashed lines indicate the identification).

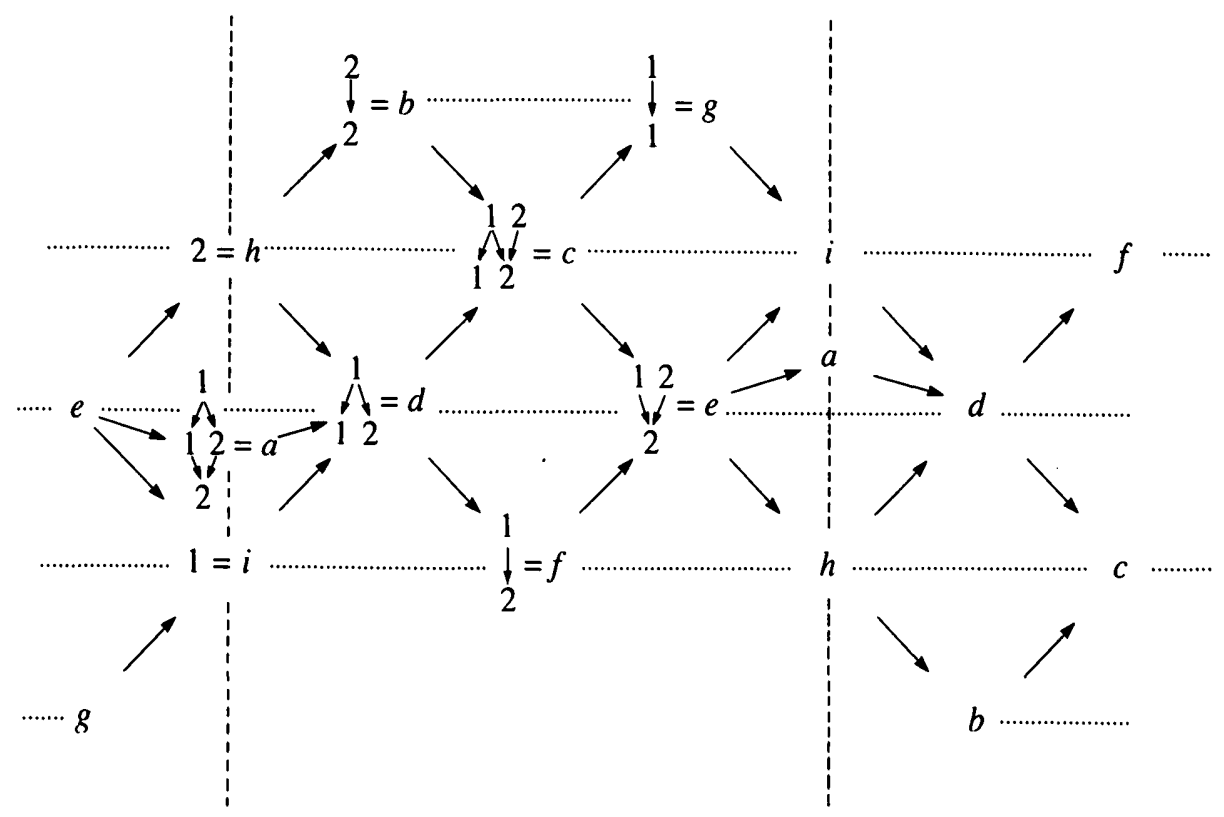

Denoting the simple modules of the Auslander algebra $A=\operatorname{End}_{R}(a \oplus b \oplus \ldots \oplus i)$ corresponding to the indecomposable $R$-module $x$ again by $x$, we see readily that $A_{A}=P_{a} \oplus P_{b} \oplus \ldots \oplus P_{i}$, where
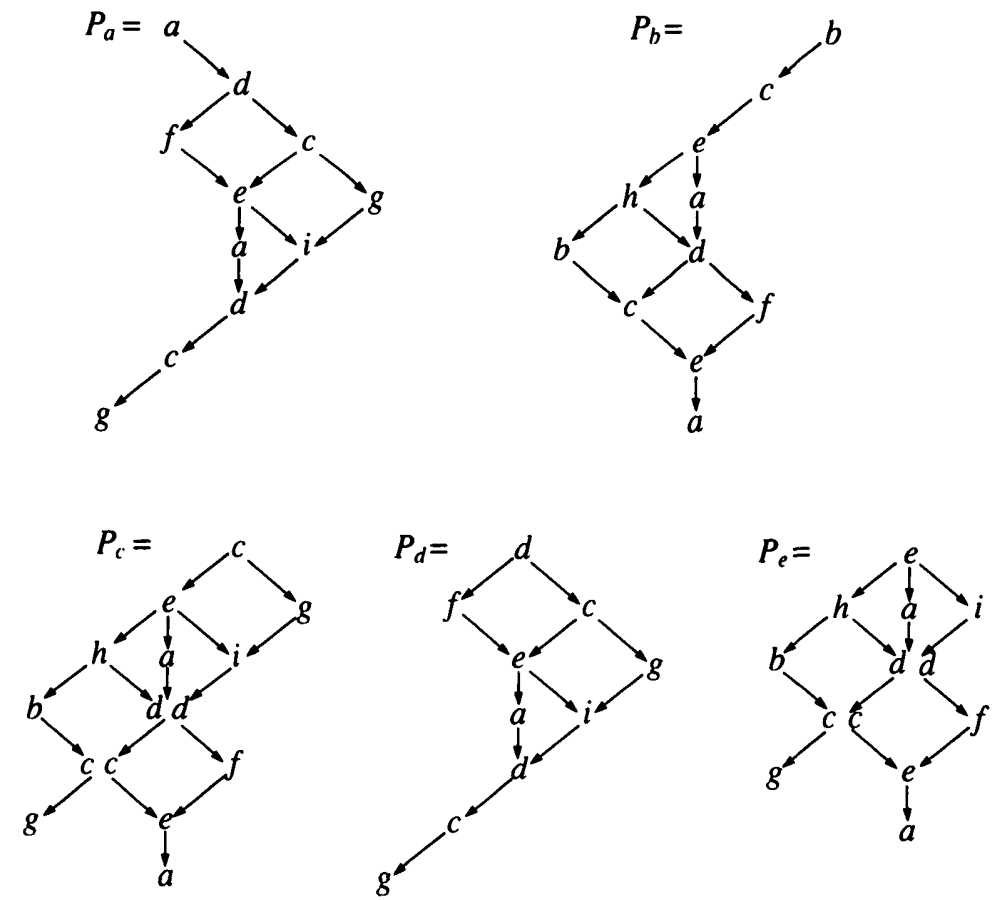

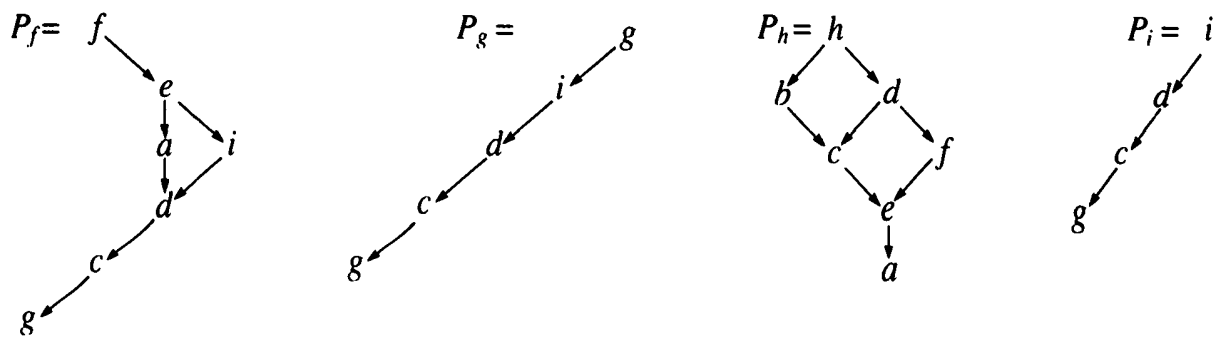

Now, consider the following splitting filtrations of ind $R$.

(i) The Rojter filtration

$$
\begin{aligned}
\varnothing \subset\{h, i\} \subset\{d, h, i\} & \subset\{b, d, f, g, h, i\} \subset\{b, c, d, f, g, h, i\} \\
& \subset\{b, c, d, e, f, g, h, i\} \subset \text { ind } R .
\end{aligned}
$$

(ii) The dual Rojter filtration

$$
\begin{aligned}
\varnothing \subset\{h, i\} \subset\{e, h, i\} & \subset\{b, e, f, g, h, i\} \subset\{b, c, e, f, g, h, i\} \\
& \subset\{b, c, d, e, f, g, h, i\} \subset \text { ind } R .
\end{aligned}
$$

(iii) A filtration

$$
\begin{aligned}
\varnothing \subset\{h, i\} \subset\{f, g, h, i\} & \subset\{e, f, g, h, i\} \subset\{d, e, f, g, h, i\} \subset\{c, d, e, f, g, h, i\} \\
& \subset\{b, c, d, e, f, g, h, i\} \subset \text { ind } R
\end{aligned}
$$

corresponding to the preprojective partition

$$
\mathscr{P}_{0}=\{a, b\}, \quad \mathscr{P}_{1}=\{c, d\}, \quad \mathscr{P}_{2}=\{e, f, g\}, \quad \mathscr{P}_{3}=\{h, i\} .
$$

(iv) A filtration

$$
\begin{aligned}
\varnothing \subset\{h, i\} \subset\{b, f, h, i\} & \subset\{b, d, f, h, i\} \subset\{b, d, e, f, h, i\} \subset\{b, c, d, e, f, h, i\} \\
& \subset\{b, c, d, e, f, g, h, i\} \subset \text { ind } R
\end{aligned}
$$

corresponding to the preinjective partition

$$
\mathscr{I}_{0}=\{a, g\}, \quad \mathscr{I}_{1}=\{c, e\}, \quad \mathscr{I}_{2}=\{b, d, f\}, \quad \mathscr{I}_{3}=\{h, i\} .
$$

In each case, the heredity chain, as well as the sequence of the (residue) quasihereditary algebras is easy to describe. For example, describing the (residue) quasihereditary algebras as (right) modules, one gets in (iii) the following sequence:

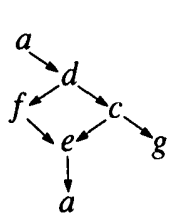<smiles></smiles>

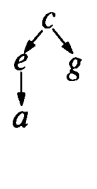

$\oplus$<smiles>O=C1C2CCC1C2(Cl)Cl</smiles>

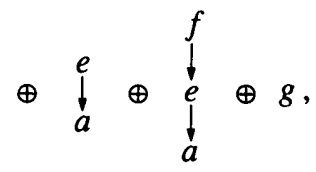

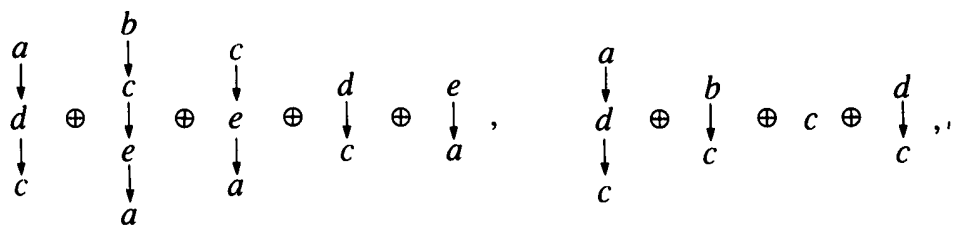

$$
a \oplus \underset{c}{b} \oplus c, \quad a \oplus b, \quad a
$$




\section{References}

1. M. Auslander and S. Smalø, 'Preprojective modules over artin algebras', J. Algebra 66 (1980) 62-122.

2. E. Cline, B. Parshall and L. Scott, 'Algebraic stratification in representation categories', $J$. Algebra to appear.

3. V. DLAB and C. M. RiNGeL, 'Quasi-hereditary algebras', Illinois J. Math. to appear.

4. P. GABRIEL, 'Indecomposable representations II', Symposia Matematica 11 (Istituto Nationale di alta Matematica, Roma, 1973) 81-104.

5. B. PARSHALl and L. 'SCOTt, 'Derived categories, quasi-hereditary algebras and algebraic groups', Proceedings of Ottawa-Moosonee Workshop in Algebra, Carleton University Notes 3 (1988).

6. A. V. ROJTER, 'Unbounded dimensionality of indecomposable representations of an algebra with an infinite number of indecomposable representations', Izv. Akad. Nauk SSSR. Ser Mat. 32 (1968) 1275-1283; Math. USSR Izv. 2 (1968) 1223-1230.

7. L. ScorT, 'Simulating algebraic geometry with algebras. I: The algebraic theory of derived categories', Proceedings of Symposia in Pure Mathematics 47 (American Mathematical Society, Providence, 1988) 271-281.

Department of Mathematics and Statistics

Carleton University

Ottawa

Canada KIS 5B6
Fakultät für Mathematik

Universität Bielefeld

D-4800 Bielefeld

West Germany 